\title{
Nb3Sn High Field Magnets for the High Luminosity LHC Upgrade Project
}

\author{
Giorgio Ambrosio
}

\begin{abstract}
The High Luminosity upgrade of the Large Hadron Collider at CERN requires a new generation of high field superconducting magnets. High field large aperture quadrupoles (MQXF) are needed for the low-beta triplets close to the ATLAS and CMS detectors, and high field two-in-one dipoles (11 T dipoles) are needed to make room for additional collimation. The MQXF quadrupoles, with a field gradient of $140 \mathrm{~T} / \mathrm{m}$ in $150 \mathrm{~mm}$ aperture, have a peak coil field of $12.1 \mathrm{~T}$ at nominal current. The $11 \mathrm{~T}$ dipoles, with an aperture of $60 \mathrm{~mm}$, have a peak coil field of 11.6 $\mathrm{T}$ at nominal current. Both magnets require $\mathrm{Nb}_{3} \mathrm{Sn}$ conductor and are the first applications of this superconductor to actual accelerator magnets.

Collaboration between the US LARP (LHC Accelerator Research Program) and CERN is developing the MQXF magnets, whereas the $11 \mathrm{~T}$ dipole magnets are being developed by CERN and Fermilab.

This paper reviews the status of $\mathrm{Nb}_{3} \mathrm{Sn}$ technology for accelerator magnets, discusses its main challenges, and discusses how the MQXF and $11 \mathrm{~T}$ designs are addressing them.
\end{abstract}

Index Terms- High field magnets, HiLumi, LHC, Superconducting magnets.

\section{INTRODUCTION}

$\mathrm{D}^{\mathrm{s}}$ ESPITE the discovery of superconductivity in $\mathrm{Nb}_{3} \mathrm{Sn}$ is 60 years old, $\mathrm{Nb}_{3} \mathrm{Sn}$ accelerator magnets are not yet a reality in High Energy Physics (HEP) applications [1]. The High Luminosity upgrade [2] of the Large Hadron Collider at CERN is about to change this landscape by relying on $\mathrm{Nb}_{3} \mathrm{Sn}$ for the low-beta quadrupoles (MQXF) [3] in ATLAS and CMS interaction regions, and for high field two-in-one dipoles (11T) [4] to make room for additional collimators by replacing some NbTi dipoles.

The main challenge that has prevented the use of $\mathrm{Nb}_{3} \mathrm{Sn}$ in HEP accelerator magnets is its brittleness, which can cause permanent degradation under very low intrinsic strain [5].

Different techniques have been developed to address this issue. The most relevant to HEP applications are the "Reactand-Wind" and the "Wind-and-React" techniques. In both techniques the conductor undergoes a heat treatment (reaction) to create the superconductor after the Rutherford cable is made. The reaction can be performed before coil winding

Template version 5.2, January 6, 2014.

Manuscript received August 12, 2014. Work supported by the US Department of Energy through the US LHC Accelerator Research Program (LARP) and by the European Commission FP7 HiLumi LHC - Grant Agreement 284404.

Giorgio Ambrosio is with Fermi National Accelerator Laboratory, Batavia, IL 60510 USA; phone: 630-840-2297; fax: 630-840-8079; e-mail: giorgioa@fnal.gov.
TABLE I

MQXF AND 11T MAIN PARAMETERS

\begin{tabular}{lccc}
\hline \multicolumn{1}{c}{ Parameter } & Unit & MQXF & $11 \mathrm{~T}$ \\
\hline N. of poles & & 4 & 2 \\
Coil aperture & $\mathrm{mm}$ & 150 & 60 \\
Magnetic length ${ }^{\dagger}$ & $\mathrm{m}$ & $2 * 4 / 6.8$ & 5.3 \\
N. of layers & & 2 & 2 \\
N. of turns per layer & $\mathrm{K}$ & $22 / 28$ & $22 / 34$ \\
Operation temperature & $\mathrm{T} / \mathrm{m}-\mathrm{T}$ & 140 & 1.9 \\
Nominal gradient/field & $\mathrm{kA}$ & 17.5 & 11.2 \\
Nominal current & $\mathrm{T}$ & 12.1 & 11.85 \\
Peak field at nom. current & & $20 \%$ & $19 \%$ \\
Margin on load line & $\mathrm{MJ} / \mathrm{m}$ & 1.3 & 0.97 \\
Stored energy at nom. current & $\mathrm{mH} / \mathrm{m}$ & 8.2 & 12 \\
Differential induct. at nom. cur. & $\mathrm{MN} / \mathrm{m}$ & 3.9 & 1.6 \\
Force on midplane per half coil & $\mathrm{m}$ &
\end{tabular}

${ }^{\dagger}$ MQXF Q1 and Q3 are made of two magnets in one helium vessel for a total magnetic length of 8m; MQXF Q2a and Q2b are made of a single magnet each with $6.8 \mathrm{~m}$ magnetic length.

("React-and-Wind") if it involves only gentle bending as in a Common-Coil design [6]. It must be performed after the winding ("Wind-and-React") in all other cases including with the shell-type coils to be used in the MQXF and 11T magnets.

The Wind-and-React technique addresses the brittleness issue during winding, but it generates other issues because it limits the options for conductor and coil parts insulation since the materials involved must be able to withstand the coil heat treatment (typically up to temperatures around $650{ }^{\circ} \mathrm{C}$ ).

In this paper we will look at the main challenges related to design and fabrication of $\mathrm{Nb}_{3} \mathrm{Sn}$ accelerator magnets using shell-type coils, and we will discuss the solutions developed for the MQXF and 11T magnets. Other designs, such as blocktype coils with stress management [7] or without it [8],[9], and canted cosine theta coils [10] are in an earlier stage of R\&D and are not considered in this paper. The MQXF quadrupoles are being developed by collaboration between the CERN HLLHC project [11] and the US LHC Accelerator Research Program (LARP) [12]. The 11T Dipole is being developed by collaboration between CERN HiLumi project and Fermilab High Field Magnet program [13]. The main features of MQXF and $11 \mathrm{~T}$ magnets are shown in Table I.

\section{CONDUCTOR}

The main challenges for the conductor to be used in $\mathrm{Nb}_{3} \mathrm{Sn}$ accelerator magnets affect both strands and cables. The strand should allow for:

- High critical current density $\left(\mathrm{J}_{\mathrm{c}}\right)$ in order to fully exploit the advantages of $\mathrm{Nb}_{3} \mathrm{Sn}$ over $\mathrm{NbTi}$, which is easier to use.

- Sufficient strain sensitivity margin in order to avoid 
degradation caused by coil handling, magnet assembly or operation.

- Small sub-element size (closely related to the effective filament diameter after reaction) in order to reduce low field magnetization, and to avoid thermo-magnetic instability and its possible enhancement due to local defects, damage, or other causes [14].

- Sufficient residual resistivity ratio (RRR) of the stabilizing copper after heat treatment, which helps to prevent both thermo-magnetic and self-field instability.

- Small degradation of $J_{c}$ and RRR due to cabling (this property has to do with both the strand features and the cable design).

Other challenges for the Rutherford cable are:

- Sufficient mechanical stability (i.e. no strands pushed out of the cable envelope ("popped strands") during coil end winding.

- Adequate inter-strand resistance in order to avoid excessive dynamic effects (such as possible quench during current ramp down and large harmonic variations due to eddy currents), while still assuring sufficient cable stability.

The strand choice is a tradeoff based on these challenges, magnet requirements (for instance regarding low field magnetization), and vendor constrains (for instance, the larger the number of sub-elements the more difficult the strand manufacturing, with higher risks of low performance and short piece length). This choice is not straightforward because of the limited experience in high volume production of high $\mathrm{J}_{\mathrm{c}}$, small filament, $\mathrm{Nb}_{3} \mathrm{Sn}$ strands, and because of the limited number of prototypes made with the same features for evaluating conductor requirements. A difficult question for instance is "what is the maximum acceptable sub-element diameter for a given application?” Tests performed by LARP on short $90 \mathrm{~mm}$ aperture magnets (TQ series) have shown limited performance [TQS02] at $1.9 \mathrm{~K}$ with strands having $64 \mathrm{um}$ sub-element diameter $\left(\mathrm{d}_{\mathrm{se}}\right)$, whereas the last TQ model with $45 \mathrm{um} \mathrm{d}_{\mathrm{se}}$ showed the expected temperature dependence [TQS03]. Nonetheless a long model magnet (LQS03) with the same coil design and conductor used by TQS03 did not show the expected temperature dependence [17]. Low RRR (50) and low prestress triggering self-field instability have been pointed to as likely causes. Some authors [18] claim that the self-field instability had a significant role also in the different behavior between TQS02 and TQS03.

In order to address the above mentioned question, many authors have developed models [19]-[21] and have performed tests on strands [22]-[25] and cables [26]-[27]. Nonetheless magnet tests have shown a level of complexity not yet reproduced in conductor tests. Examples of this complex behavior are: (i) the decrease of the instability threshold seen by testing the same magnet or coil under increasing prestress [28]; and (ii) the "enhanced instability" mechanism [14] where damage or another issue caused a local decrease of the instability threshold in a single coil. Analysis of the issues seen during the tests of these and other $\mathrm{Nb}_{3} \mathrm{Sn}$ magnets suggests that smaller subelements may allow a greater margin against known and unknown issues. On the other hand, there may be more risks posed by the production of strands with more subelements. The strand choice for $\mathrm{Nb}_{3} \mathrm{Sn}$ accelerator magnets is therefore necessarily subject to a risk mitigation analysis.

Also the cable design is subject to difficult tradeoffs if the cable contains a large number of strands. The compaction needed to preserve the mechanical stability of the cable (i.e. no risk of strands popping out during winding) causes significant deformation of the strands at the edges of cables containing a high number of strands. This deformation may break the barrier around each sub-element (Fig. 1) causing degradation of critical current $\left(\mathrm{I}_{\mathrm{c}}\right), \mathrm{RRR}$, and the stability threshold. The latter is more dangerous and appears more significant that the $I_{c}$ degradation, which therefore is not a good test for establishing an acceptable level of strand deformation. Several authors [29] are focusing on the analysis of local RRR degradation around the cable edges of extracted strands in order to establish acceptance criteria. This work is very promising especially since it can be related to experimental studies of RRR degradation and instability [30]. Nonetheless, as previously mentioned, there are some open questions about the relations between conductor measurements and magnet performances in a series production (for instance: how much margin is needed in order to achieve a sustainable coil success rate?). It is anticipated that the MQXF and 11T projects will generate sets of data for addressing these questions. Until then, the magnet designers are left with a difficult risk management exercise.

The main features of the MQXF and 11T conductors are shown in Table II. It should be noted that both projects are developing two strand options with similar features: (i) the Restacked Rod Process (RRP) wire made by Oxford Superconducting Technology (OST) with 132 superconducting subelements (hollow filaments after the reaction) out of 169 subelements for both projects; and (ii) the Powder in Tube (PIT) wire by Bruker-EAS with 192 filaments for MQXF and 120 filaments for 11T. Developing two options avoids the risk of a single source, and developing similar strand designs for both projects increases the volume

TABLE II

STRAND AND CABLE PARAMETERS

\begin{tabular}{lccc}
\hline \hline Parameter & Unit & MQXF & $11 \mathrm{~T}$ \\
\hline Strand diameter & $\mathrm{mm}$ & 0.85 & 0.7 \\
Fabrication process & & RRP, PIT & RRP, PIT \\
Number of filaments & & 132,192 & 132,120 \\
Nominal sub-element diameter & $\mathrm{um}$ & $<50$ & $<45$ \\
RRR after full heat treatment & & $>150$ & $>100$ \\
Cu/non-Cu & & 1.2 & 1.15 \\
Minimum Ic (12 T, 4.222 K) ${ }^{\dagger}$ & $\mathrm{A}$ & & 438 \\
Minimum Ic (15 T, 4.222 K) & & 361 & \\
Number of strands & $\mathrm{A}$ & 40 & 40 \\
Cabling degradation & & $<5$ & $<5$ \\
Cable bare width & $\%$ & 18.15 & 14.70 \\
Cable bare mid-thickness & $\mathrm{mm}$ & 1.525 & 1.25 \\
Keystone angle & $\mathrm{mm}$ & 0.55 & 0.79 \\
\hline \hline
\end{tabular}

${ }^{\dagger}$ Without self-field correction. 
fabricated during the prototyping phase, which in turn reduces production risk. The subelement size in the 132/169 RRP strand with $0.85 \mathrm{~mm}$ diameter is equal to the subelement size (50 um) in the 108/127 RRP strand with $0.778 \mathrm{~mm}$ diameter, which was used in the very successful HQ02 magnet [31].

Another important optimization has to do with the strand $J_{c}$ and RRR after heat treatment (HT). The temperature and duration of the last HT plateau can be adjusted in order to achieve the highest $\mathrm{J}_{\mathrm{c}}$ (for instance above $3000 \mathrm{~A} / \mathrm{mm}^{2}$ at 4.2 $\mathrm{K}$ and $12 \mathrm{~T}$ in RRP wires) at the expense of limited RRR (typically below 100), or to preserve high RRR with some loss of $\mathrm{J}_{\mathrm{c}}$. A significant step forward was recently made by reducing the tin content of RRP wires, allowing both high $\mathrm{J}_{\mathrm{c}}$ and high RRR [32], [33].

The strain sensitivity of RRP strands was significantly improved by changing the ternary addition from tantalum to titanium [5], which moved the irreversible intrinsic strain limit from about zero to $\sim 0.3 \%$.

Both MQXF and 11T cables have 40 strands. This number corresponds to the maximum number of spools that can be used by the CERN cabling machine, and is driven by the need of large coils, which improve stress distribution and quench protection, still within the limit of two-layer coils. This large number of strands makes the design of keystoned cables quite challenging, and while both projects have found a balance between cable compaction and strand deformation, the resulting cables have been characterized by marginal mechanical stability. In the next session we will discuss the solutions developed for winding coils using these cables.

Both cables have a 25 um thick stainless steel core for reducing dynamic effects caused by the low inter-strand crossover resistance after reaction. The core in the $11 \mathrm{~T}$ cable covers almost the entire internal surface of the cable; whereas in the QXF cable the core covers $72-73 \%$ of the internal surface. The HQ02 quadrupole [31] demonstrated acceptable dynamic performance with a core covering $60 \%$ of its internal surface.

The US contribution (LARP) to the MQXF collaboration initiated the development of the MQXF cable using 108/127 RRP strands by OST. Tests of strands extracted from MQXF prototype cables and from cables for short model coils have

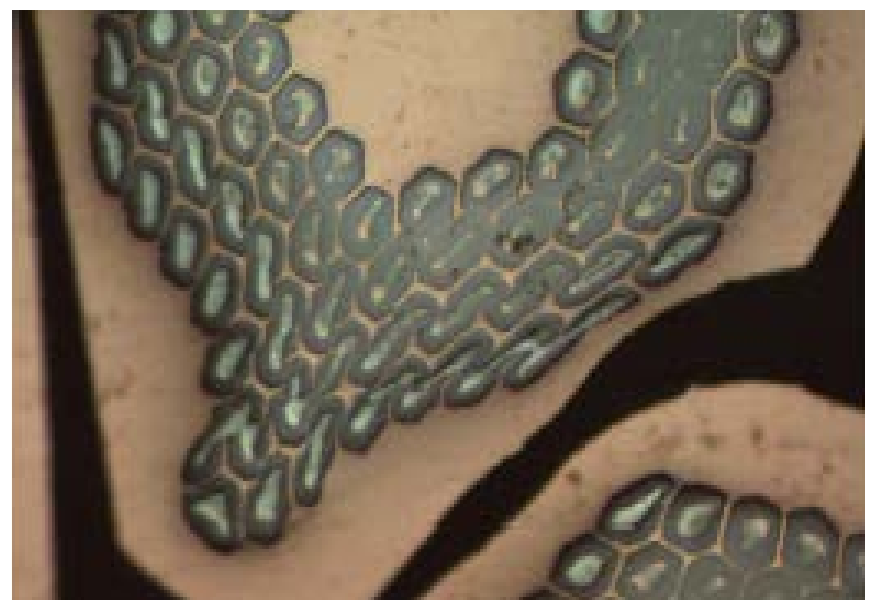

Fig. 1. Micrograph of MQXF cable edge showing sheared sub-elements in a strand. (Courtesy D. Dietderich) shown very small $I_{c}$ degradation [33]. Micrographs of cable edges have shown a larger number of damaged sub-elements than in previous LARP magnets (Fig 1) [33]. Nonetheless, preliminary study of local RRR around cable edges has shown acceptable values (above 150 for 48 hour reaction at $650{ }^{\circ} \mathrm{C}$ ). Procurement of 132/169 RRP strand is in progress and tests on nine billets received by LARP and ten billets received by CERN have shown that the heat treatment can be adjusted to exceed all requirements [34].

Tests of $11 \mathrm{~T}$ strands and cables [26], [27] have shown that the conductor is on track for meeting all requirements.

\section{MAgnetiC Design AND Operation REQUiREMENTS}

The design and fabrication of accelerator-quality $\mathrm{Nb}_{3} \mathrm{Sn}$ magnets faces several challenges, some of which depend on the application.

- Field quality at injection: $\mathrm{Nb}_{3} \mathrm{Sn}$ strands have larger filaments than $\mathrm{NbTi}$ strands, resulting in larger magnetization affecting harmonics at injection. This issue is more severe for the $11 \mathrm{~T}$ dipoles, which will be series powered with standard LHC dipoles and should have similar harmonic content. In order to mitigate this issue, the RRP strand option was changed from the original 108/127 design [2] to the present 132/169 design; the minimum current may be lowered from 350 A to $100 \mathrm{~A}$; and passive magnetic shims may be installed in the coil aperture [2]. Impact on MQXF and a detailed analysis of this issue in high-field $\mathrm{Nb}_{3} \mathrm{Sn}$ accelerator magnets can be found in [35].

- Effect of iron saturation on field quality: the high field of these magnets causes large-scale saturation of the iron yoke. The impact of iron saturation on field quality is mitigated by shaping the iron and adding holes [4].

- Impact of dynamic effects on field quality: eddy current loops may distort field quality in unpredictable ways if the inter-strand contact resistance varies by orders of magnitude as was seen with several past $\mathrm{Nb}_{3} \mathrm{Sn}$ magnets. The introduction of a 25 um stainless steel core in the Rutherford cables solved this issue [36] and is used by both projects.

- Field quality reproducibility and not allowed harmonics: there is little experience pertaining to reproducibility in a series of $\mathrm{Nb}_{3} \mathrm{Sn}$ magnets. The MQXF is planning to test magnetic shims located in the gaps used by bladders during magnet assembly. Simulations have shown that this technique can provide correction for low order harmonics up to a few units [37].

- Radiation damage and energy deposition: this is a special concern for the MQXF quadrupoles, which will be exposed to debris from the interaction points. The MQXF aperture was set to $150 \mathrm{~mm}$ in order to allow for tungsten absorbers inside each magnet aperture. The absorbers are thicker on coil midplanes (16 mm for Q1 and $6 \mathrm{~mm}$ for Q2 and Q3) where the magnetic field focuses charged particles. Simulations have shown that with these absorbers the peak integrated dose does not exceed 
25 MGy for $3000 \mathrm{fb}^{-1}$ integrated luminosity; and the peak energy deposition is below $2 \mathrm{~mW} / \mathrm{cm}^{-3}$ with $510^{34} \mathrm{~cm}^{-2} \mathrm{~s}^{-1}$ peak luminosity [38].

\section{COIL FABRICATION}

The fabrication of Wind-and-React, shell-type, $\mathrm{Nb}_{3} \mathrm{Sn}$ coils to be used in accelerator magnets faces several challenges:

- The winding of Rutherford cables with limited mechanical stability, which increases the risk of "popped strands" during coil-end winding.

- The coil handling is tricky until potting, since insulation with B-stage or other glue cannot be used because of the heat treatment.

- All coil parts installed before reaction (for instance: end spacers, pole pieces, cable insulation, and layer-layer insulation) should be able to withstand the heat treatment; and the metallic parts should be electrically insulated from the conductor in order to avoid shorts.

- The reaction tooling and the metallic coil parts should be designed in order to accommodate the different thermal expansions and the coil dimensional changes which occur during reaction; on the other hand, the reaction tooling should be sufficiently tight to assure the correct conductor placement for field quality.

- Tooling and processes for coil handling should prevent excessive conductor strain after reaction.

- The coils should be potted with epoxy resin (or similar materials) in order to facilitate magnet assembly and avoid stress concentrations. Attempts to avoid this step have resulted in mediocre performance [39].

- The materials used for coil potting should be compatible with the expected radiation dose and should not limit heat extraction.

- The heaters to be used for quench protection should be compatible with the fabrication process and should not compromise heat extraction.

The MQXF quadrupoles and the $11 \mathrm{~T}$ dipoles share many common features for addressing these challenges, but there are some noticeable differences. There are also some differences between the $11 \mathrm{~T}$ models built at FNAL and those built at CERN.

Among the common features we should mention the following: (i) coils are wound using the double-pancake technique without inter-layer splice; (ii) after winding, each layer is painted with a ceramic binder (CTD-1202X) and cured at $150{ }^{\circ} \mathrm{C}$ so that it becomes a solid object; (iii) the reaction and potting fixtures are made of precise blocks and plates so that the fixtures can be assembled and disassembled around the coil, reducing to a minimum the risk of damaging the coil; (iv) coils are potted with CTD-101K epoxy resin, which preserves good structural and electrical properties after 25 MGy irradiation dose [40] (MQXF requirement). These features have been pioneered by FNAL HFM program [41] and improved by LARP [42] through the development and fabrication of more than 50 short $(\sim 1 \mathrm{~m})$ coils and 20 long $(\sim 3.5 \mathrm{~m})$ coils. Some authors [43] have pointed out that the presence of the binder on the fiberglass (in the cable insulation and in the layer-layer insulation) causes embrittlement of the fibers after reaction. Nonetheless, at this point in the coil fabrication process the fiberglass is acting as a spacer (i.e. with no structural requirements) and the subsequent potting freezes the fiberglass in place. The excellent performance of coils built using this technology [16], [44], [31] shows that the technology can meet the requirements of the MQXF and 11T projects.

There is a small difference in the curing process between MQXF and 11T coils. The $11 \mathrm{~T}$ coils are cured in a cavity with azimuthal dimension slightly smaller than the nominal coil size in order to allow for azimuthal coil growth during reaction. In contrast, the curing cavity of the MQXF coils nominal coil size and room for azimuthal expansion is provided by design (i.e. the coil size is computed taking into account the fact that the reacted cable is larger than the nonreacted cable) [45].

LARP is addressing the limited mechanical stability of the MQXF cable during winding by using a special tool, which applies a tight fit to the insulated cable, and by reinforcing the cable insulation in the ends (a small amount of binder is painted and cured on each turn just before end winding). The special tool has been successfully developed at LBNL for HQ02 coil fabrication (although some popped strands had to be fixed by hand). The binder technique was successfully used for the 11T coils wound by FNAL.

The MQXF and 11T teams are exploring different solutions for cable insulation. The MQXF cable has 145 um thick braided insulation made of S2-glass. The FNAL $11 \mathrm{~T}$ cable is wrapped by a 75 um thick E-glass tape with $50 \%$ overlap. The CERN 11T cable is insulated by a C-shaped 80 um thick mica foil and by 75 um thick S2-glass braided around it (the insulation thickness after reaction is about $110 \mathrm{um}$ ).

All coils use stainless steel end parts and saddles, and the pole pieces are made of Ti-6Al-4V. Gaps among the pole pieces are preserved by temporary shims during winding and curing. These gaps, which are closed or almost closed after reaction, avoid coil strain due to cable shrinkage (annealing) and different contraction between coil and poles at the end of the reaction cycle.

In the MQXF and FNAL 11T coils the pole pieces are glued to the coil during potting. On the contrary, CERN 11T coils employ a removable pole in order to allow adjustments to the pre-stress of pole turns. A filler wedge on the outer layer is potted with the coil to match the azimuthal size of the inner layer.

The end parts for $11 \mathrm{~T}$ coils have been designed using ROXIE. The MQXF end parts have been designed using BEND (by LARP) and ROXIE (by CERN) [46]. Different features have been introduced to give some "flexibility" to the end parts in order to prevent insulation damage because during winding the turns in the coil ends have a tendency to separate from the pole and the mandrel: $11 \mathrm{~T}$ end parts have flexible legs; MQXF end parts have accordion-style slits in the center. Coil fabrication has shown that both features are effective in preventing insulation damage. 


\section{Support StRucture AND MAGNET ASSEMBLY}

The design of the support structures for these magnets is facing huge challenges because these structures should withstand forces of some $\mathrm{MN} / \mathrm{m}$ and preserve the nominal coil shape with tight tolerances without allowing excessive stress in the brittle $\mathrm{Nb}_{3} \mathrm{Sn}$ coils at any step of assembly, cooldown or operation.

From this point of view the MQXF and $11 \mathrm{~T}$ are exploring different solutions with one common point: both designs attempt to maximize the coil width with a two-layer coil layout by using 40 strands (maximum number for CERN cabling machine). In the MQXF, prestress and support are provided by an aluminum shell preloaded by the use of bladders and keys. This concept, developed at LBNL for RD3 [47] has been used by all of the latest LARP magnet series: the $120 \mathrm{~mm}$ aperture, $1 \mathrm{~m}$ long HQ quadrupoles, and the $90 \mathrm{~mm}$ aperture, $3.6 \mathrm{~m}$ long LQ quadrupoles. 2D analysis has shown that MQXF coil peak stress remains below $170 \mathrm{MPa}$ during all steps of assembly and operation [48]. This value is comparable with the coil peak stress in HQ02 that reached 173 MPa. A preliminary 3D stress analysis of MQXF [48] has shown higher values possibly caused by the discontinuity of the yoke in the ends (in order to keep the peak field in the straight section) and by the shell axial contraction. These features were present also in the HQ02 magnet and did not limit its excellent performance.

In the two-in-one $11 \mathrm{~T}$ dipole, separate stainless-steel collars are used for each aperture. In the removable-pole design the collars provide full prestress and support (with some assistance from the yoke resulting from a small interference around the midplane), whereas in the integrated-pole design, additional prestress is provided by the skin during cooldown resulting from the vertical gap in the yoke, which should close during cooldown and remain closed during operation. In both cases 2D stress analysis showed peak stress in the coil below $150 \mathrm{MPa}$ during all steps of assembly and operation [4].

The differences between these structures make any comparison quite difficult. A detailed analysis is beyond the scope of this paper and can be found elsewhere [4], [48]. Here we stress that the different choices made by the MQXF and $11 \mathrm{~T}$ designers show the strength of each concept. The Al-shell based design allows moderate and well controlled coil preload at room temperature because of the preload increase during

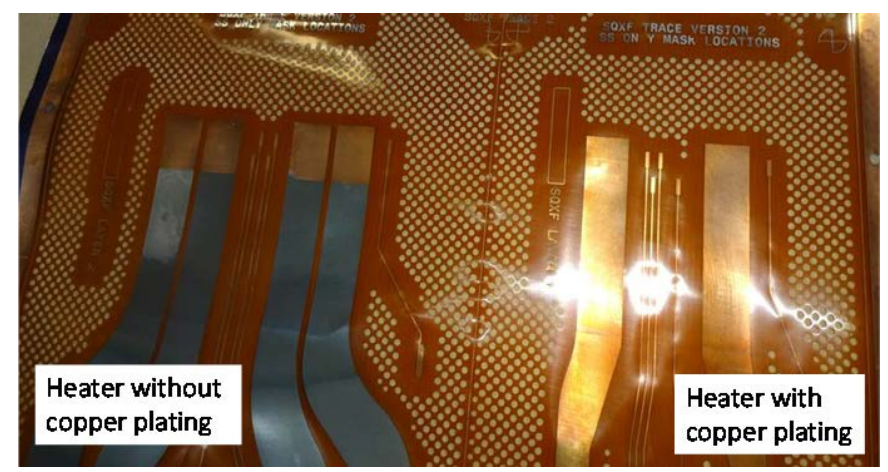

Fig. 2. Trace for SQXF coil showing copper plating on some heaters (on the right), and perforations of the polyimide for porosity to helium.

(Courtesy of J.C. Perez). cooldown. This is advantageous for magnets with very high magnetic forces. The collar-based design benefits from decades of SC accelerator magnet development using this concept and is very advantageous for two-in-one magnets.

Even if the choice of the structure is well matched with the application there are significant challenges for each design. The MQXF should demonstrate that the excellent performance reached in short models [31] can be reproduced in long magnets during a mini-series production. The LARP Long Quadrupole successfully addressed several scale-up challenges (for instance the use of a segmented shell to accommodate the different longitudinal thermal contraction of the iron yoke and aluminum shell) but not all of them (for instance the LQ coils did not have alignment features).

The CERN-design $11 \mathrm{~T}$ should demonstrate control of the coil-pole interface during all stages of assembly and operation of two-in-one dipoles. Similarly the FNAL-design 11T should demonstrate control of the yoke gap during all stages of assembly and operation.

\section{QUENCH PROTECTION}

The quench protection of these magnets is particularly challenging because of the high operating current density and energy density (for instance two times larger than in LHC magnets) [49]. The coil potting also prevents the possible beneficial effect of direct contact between conductor and superfluid helium.

Because of the brittleness of the $\mathrm{Nb}_{3} \mathrm{Sn}$ conductor, a goal of quench protection is to prevent stresses and strain that could degrade the conductor. Degradation and detraining may come from the coil/structure interaction. Studies performed on the HQ02 magnet with different preload levels (medium in HQ02a, and high in HQ02b) demonstrated significantly less detraining in HQ02b during high-temperature quenches (quenches with a hot spot temperature purposely higher than normal). The high-temperature quenches in HQ02b had to be stopped due to limited availability of the CERN test facility; nonetheless, they showed promising results including a quench above $400 \mathrm{~K}$ without large degradation [31]. Analysis of high-temperature quenches during tests of cables, a small racetrack, and a 90-mm aperture quadrupole has shown that the maximum acceptable temperature in $\mathrm{Nb}_{3} \mathrm{Sn}$ magnets should be lower than the glass transition temperature of its epoxy (386 K for CTD-101K) [50].

The best strategy for protecting long $\mathrm{Nb}_{3} \mathrm{Sn}$ magnets (with high inductance) is to distribute the stored energy onto the whole coil volume as soon as possible. Therefore both projects are planning to use protection heaters and are facing a challenging tradeoff between high electrical strength and low thermal diffusion time. The MQXF team is using heaters embedded in the coil during potting. The heaters are photoetched on a 25-um thick stainless steel foil glued to a 50-um polyimide foil (Fig. 2) named "trace" because it also carries the signals from the voltage taps to external wires [51]. The $11 \mathrm{~T}$ team is using heaters made of stainless steel strips located between ground plane insulation layers.

The first complete simulation of quenches in MQXF 
assumed heaters only in the outer layer and faced difficulties in keeping the hot spot temperature below $350 \mathrm{~K}$ [52] even without any heater failure. A subsequent effort aimed at improving the MQXF quench protection without magnet design changes achieved this goal by several developments. First, comparison of simulations with actual quench data demonstrated that dynamic effects (mostly inter-filament eddy currents) help with extracting energy faster than previously assumed [53]. Second, the development of copper cladding on the heaters embedded in the traces helped in reducing the time needed to completely quench long coils. Third, special quench heaters for the inner layer have been developed. These heaters should be set on the inner surface of the coil's inner layer before potting in order to be glued to the coil aperture by the epoxy. This solution was tested on several LARP magnets (LQ and HQ models) and an issue was identified involving the partial delamination of the trace from the coil causing breakage of the heater on a few occasions [54]. This issue appears to be caused by superfluid helium trapped in the coil or between coil and trace during a quench and affects only heaters on the inner layer (IL) where there is no structural element preventing the delamination. The designs under development for the MQXF IL traces are attempting to minimize the heater coverage by using copper cladding in order to render most of the trace surface porous to helium owing to a pattern of small holes (Fig. 2) or large cut-outs. Updated MQXF quench simulations taking into account these improvements have shown that the hot spot temperature remains below $300 \mathrm{~K}$ even using only half the available heaters, so that redundancy can be provided by two separate circuits [53].

Further improvement to the MQXF quench protection may result from the use of a Coupling-Loss Induced Quench system (CLIQ), which induces coil quench by inter-filament coupling losses generated by the magnetic field change generated when a large capacitance is discharged in the coil winding [55]. A CLIQ system was successfully used to protect the HQ02 magnet during a test at CERN [56], demonstrating that it is a very promising option for MQXF protection. CLIQ works very efficiently on the inner layers and therefore it is a good match with heaters on the outer layers and could provide perfect redundancy. Nonetheless some open questions have to be addressed; for instance, its efficiency in protecting long magnets and system compatibility with LHC operation. The test facilities that are being upgraded for MQXF short and long prototypes will be compatible with CLIQ systems in order to validate them.

The quench protection of the two-in-one $11 \mathrm{~T}$ dipole is based on high-efficiency protection heaters set in the ground insulation on the outer surface of each coil [4]. Several measurements have been performed during tests of the first short model coils; for instance, layer-layer quench propagation [57], and efficiency of copper plated heaters [58]). The test of a single coil in mirror structure made it possible to add instrumentation on the coil mid-plane for direct measurement of quench propagation velocities and hot-spot temperature [59].

\section{STATUS AND PLANS}

At the time this paper was submitted the QXF collaboration has completed the fabrication of short practice coils, is starting the assembly of a "mirror" structure that will be used to test the first SQXF coil at the beginning of 2015, and has started coil fabrication and structure procurement for the first short quadrupole model (SQXF1) with $1.2 \mathrm{~m}$ magnetic length. It is expected that SQXF1 will be tested in summer 2015, whereas the fabrication of full length (4 m magnetic length) coils for the Q1 and Q3 prototypes is planned to begin in 2015, with the aim of testing the first full length prototype by summer 2016 . Meanwhile the LARP collaboration is reducing the risks posed by the MQXF quadrupoles by testing the same features in models with $120 \mathrm{~mm}$ aperture. The latest short model (HQ02) demonstrated the maturity of this design concept by reaching 98\% short sample limit at $4.5 \mathrm{~K}$ (HQ02a2 test at FNAL) and 95\% at $1.9 \mathrm{~K}$ (HQ02b test at CERN), exceeding $190 \mathrm{~T} / \mathrm{m}$ gradient [31].

The development of the $11 \mathrm{~T}$ dipole is more advanced. Short single coils have been successfully tested both at FNAL using an iron mirror structure [59], and at CERN adding a copper coil in a single aperture structure [58]. Three single aperture short models have been tested at FNAL. The last two short models (MBHSP02/3) exceed the target field reaching 11.7 and 11.2 $\mathrm{T}$ [61]. The training of MBHSP03 was stopped out of concerns that the coils may be damaged because of low prestress. MBHSP02 was affected by quenches when holding a constant current for several minutes close to the operating current. MBHSP03 did not exhibit this issue. The two pairs of collared coils used in MBHSP02 \& 03 will be assembled in the first short two-in-one model after increasing the prestress of the MBHSP03 collared pair. The test is planned for fall 2014.

\section{CONCLUSION}

Plans for the High Luminosity upgrade of the LHC include the use of $\mathrm{Nb}_{3} \mathrm{Sn}$ magnets for the first time in a particle accelerator. The tremendous progress of this technology in the last decade has made this step possible. Nonetheless several challenges are still to be faced. The MQXF and 11T teams are addressing them, sometimes with similar solutions and sometimes exploring very different solutions. Both projects have already achieved important milestones, and the next few years will be decisive for declaring production readiness. It is anticipated that the success of these projects will provide new exciting options for the design of future accelerators.

\section{ACKNOWLEDGMENT}

The author thanks A. Ballarino, E. Barzi, S. I. Bermudez, B. Bordini, S. Caspi, G. Chlachidze, D. Dietderich, H. Felice, P. Ferracin, A. Ghosh, M. Juchno, M. Karppinen, V. Marinozzi, J.C. Perez, E. Ravaioli, G. L. Sabbi, F. Savary, M. Sorbi, E. Todesco, and A. V. Zlobin for very valuable contributions and comments. 


\section{REFERENCES}

[1] L. Rossi, L. Bottura, "Superconducting Magnets for Particle Accelerators" Reviews of Accelerator Science and Technology, Vol. 5, pp. 51-89, 2012.

[2] L. Bottura, G. de Rijk, L. Rossi, and E. Todesco, "Advanced Accelerator Magnets for Upgrading the LHC”, IEEE Trans. Appl. Supercond., 22 4002008

[3] P. Ferracin, G. Ambrosio, M. Anerella, F. Borgnolutti, R. Bossert, D. Cheng, D. R. Dietderich, H. Felice, A. Ghosh, A. Godeke, S. Izquierdo Bermudez, P. Fessia, S. Krave, M. Juchno, J. C. Perez, L. Oberli, G. Sabbi, E. Todesco, and M. Yu, "Magnet Design of the $150 \mathrm{~mm}$ Aperture Low- $\beta$ Quadrupoles for the High Luminosity LHC", IEEE Trans. Appl. Supercond., 244002306.

[4] M. Karppinen, N. Andreev, G. Apollinari, B. Auchmann, E. Barzi, R. Bossert, V. V. Kashikhin, A. Nobrega, I. Novitski, L. Rossi, D. Smekens, A. V. Zlobin, "Design of $11 \mathrm{~T}$ Twin-Aperture Nb3Sn Dipole Demonstrator Magnet for LHC Upgrades”, IEEE Trans. Appl. Supercond., 224901504.

[5] N. Cheggour, L. F. Goodrich, T. C. Stauffer , J. D. Splett , and X.F. Lu, A. K. Ghosh, G. Ambrosio, "Influence of Ta and Ti doping on the irreversible strain limit of ternary Nb3Sn superconducting wires made with restacked-rod process", Supercond. Sci. and Tech., 20, (2010).

[6] R. Gupta, "A common coil design for high field 2-in-1 accelerator magnets", Proceedings of the 1997 Particle Accelerator Conference, Vol. 3, pp. 3344-2246.

[7] A. McInturff, et al., "Current Status of the Texas A\&M Magnet R\&D Program”, IEEE Trans. Appl. Supercond., vol. 21, no. 3, pp. 1620 1623, June 2011.

[8] D. W. Cheng, S. Caspi, D. R. Dietderich, H. Felice, P. Ferracin, A. R. Hafalia, M. Marchevsky, S. Prestemon, G. Sabbi, "Design and fabrication experience with Nb3Sn block-type coils for high field accelerator dipoles”, IEEE Trans. Appl. Supercond., 234002504

[9] G. de Rijk, "The EuCARD High Field Magnet Project", IEEE Trans. Appl. Supercond., IEEE Trans. Appl. Supercond., 224301204.

[10] S. Caspi, L. Brouwer, T. Lipton, A. Hafalia Jr, S. Prestemon, D. Dietderich, H. Felice, X. Wang, E. Rochepault, A. Godeke, S. Gourlay and M. Marchevsky, "Design of a 18T Canted-Cosine-Theta Superconducting Dipole Magnet,", this conference.

[11] http://hilumilhc.web.cern.ch/HiLumiLHC/index.html

[12] http://www.uslarp.org/

[13] https://espace.cern.ch/dsdipole/default.aspx

[14] G. Ambrosio, et al., "Progress in the Long $\mathrm{Nb}_{3} \mathrm{Sn}$ Quadrupole R\&D by LARP”, IEEE Trans. Appl. Supercond., 224003804.

[15] S. Caspi, et al., "Test results of LARP Nb3Sn quadrupole magnets using a shell-based support structure (TQS)" " IEEE Trans. Appl. Supercond., vol. 19, no. 3, pp. 1221-1223, 2009.

[16] H. Felice, G. Ambrosio, M. Bajko, E. Barzi, B. Bordini, R. Bossert, S. Caspi, D. Dietderich, P. Ferracin, J. Feuvrier, A. Ghosh, A. Godeke, J. Lizarazo, L. Rossi, G. Sabbi, P. Wanderer, X. Wang and A. V. Zlobin, "Test results of TQS03: A LARP shell-based Nb3Sn quadrupole using 108/127 conductor” J. Phys.: Conf. Ser. 234 032010, 2010.

[17] G. Ambrosio, et al., "Test Results and Analysis of LQS03 Third Long Nb3Sn Quadrupole by LARP” IEEE Trans. Appl. Supercond., 23 4002204

[18] B. Bordini, M. Bajko, S. Caspi, D. Dietderich, H. Felice, P. Ferracin, L. Rossi, G. L. Sabbi, and E. Takala, "Magneto-Thermal Stability in LARP Nb3Sn TQS Magnets” IEEE Trans. Appl. Supercond., vol. 20, no. 3, pp. 274-278, Jun. 2010.

[19] V. V. Kashikhin and A. V. Zlobin, "Magnetic instabilities in strands and cables,” IEEE Trans. Appl. Supercond., vol. 15, no. 2, pp. 1621-1624, Jun. 2005.

[20] B. Bordini and B. L. Rossi, "Self field instability in high Jc strands with high copper residual resistivity ratio,” IEEE Trans. Appl. Supercond., vol. 19, no. 3, pp. 2470-2476, Jun. 2009.

[21] M.D. Sumption, "Stability in Nb3Sn Conductors; Magnetic and SelfField Instability Considerations at $4 \mathrm{~K}$ and $2 \mathrm{~K}$ " Advances in Cryogenic Engineering Materials, Volume 1219, pp. 199-207 (2010).

[22] A. Ghosh, "Effect of Copper Resistivity and Filament Size on the SelfField Instability of High-Jc Nb3Sn Strands" IEEE Trans. Appl. Supercond., 237100407.

[23] E. Barzi, et al., "Instabilities in transport current measurements of Nb3Sn strands”, IEEE Trans Appl. Supercond., vol 15, no. 2 Page 33643367, 2005.
[24] D.R. Dietderich, S.E. Bartlett, S. Caspi, P. Ferracin, S. A. Gourlay, H. C. Higley, A. F. Lietzke, S. Mattafirri, A. D. McInturff, G.L. Sabbi, R.M. Scanlan, "Correlation Between Strand Stability and Magnet Performance”, IEEE Trans Appl. Supercond., vol 15, no. 2 Page 15241528.

[25] B. Bordini et al., "Impact of the Residual Resistivity Ratio on the Stability of $\mathrm{Nb}_{3} \mathrm{Sn}$ Magnets”, IEEE Trans. Appl. Supercond., Vol. 22, No. 3, pp. 4705804, June 2012

[26] A. Wuis, B. Bordini;, A. Ballarino, L. Oberli, H. H. ten Kate, "Characterization of $\mathrm{Nb}_{3} \mathrm{Sn}$ Rutherford cables for the LHC 11 T Dipole Magnet” IEEE Trans. Appl. Supercond., 244003505.

[27] E. Barzi, D. Turrioni, and A.V. Zlobin, "Progress in Nb3Sn RRP Strand Studies and Rutherford Cable Development at FNAL", IEEE Trans. Appl. Supercond., 246000808.

[28] G. Chlachidze, et al., "The Study of Single Nb3Sn Quadrupole Coils Using a Magnetic Mirror Structure”, IEEE Trans. Appl. Supercond. Vol. 21, No. 3, pp. 1692-1695, 2011.

[29] A. Ghosh, D. Dietderich, A. Ballarino, B. Bordini, L. Oberli, private communication.

[30] E. Takala, B. Bordini, L. Rossi, "Perturbation Sensitivity of Magnetothermal Instability”, IEEE Trans. Appl. Supercond., Vol. 22, No. 6, pp. 4706106, December 2012

[31] H. Bajas, G. Ambrosio, M. Anerella, M. Bajko, R. Bossert, S. Caspi, A. Chuichiolo, G. Chlachidze, D. R. Dietderich, H. Felice, P. Ferracin, J. Feuvrier, A. K. Ghosh, A. Godeke, R. R. Hafalia, M. Marchevsky, G. Sabbi, T. Salmi, J. Schmalzle, E. Todesco, P. Wanderer, X. Wang, M. $\mathrm{Yu}, \mathrm{D}$. Cheng, "Cold Test Results of the LARP HQ02-b magnet at 1.9 $\mathrm{K}$ ", this conference.

[32] M. B. Field, Y. Zhang, H. Miao, M. Gerace and J. A. Parrell, "Optimizing Nb3Sn Conductors for High Field Applications" IEEE Trans. Appl. Supercond., Vol. 24, No. 3, pp. 6001105, June 2014.

[33] D. Dietderich, et al. "Optimization of Nb3Sn Cable for the Low- $\beta$ Quadrupoles for the High Luminosity LHC", this conference.

[34] A. Ghosh, A. Ballarino, unpublished data.

[35] X. Wang, G. Ambrosio, G. Chlachidze, E. Collings, D. R. Dietderich, J. DiMarco, A. K. Ghosh, A. Godeke, M. Marchevsky, S. O. Prestemon, G. Sabbi, M. D. Sumption, G. Velev, X. Xu, "Field errors induced by persistent current in high-field superconducting accelerator magnets", this conference.

[36] X. Wang, et al., "Multipoles Induced by Inter-Strand Coupling Currents in LARP Nb3Sn Quadrupoles", IEEE Trans. Appl. Supercond., Vol. 24, No. 3, pp. 4002607, June 2014.

[37] P. Ferracin and P. Hagen, private communication.

[38] E. Todesco, et al., "A First Baseline for the Magnets in the High Luminosity LHC Insertion Regions”, IEEE Trans. Appl. Supercond., Vol, 24, No. 3, pp. 4003305, June 2014.

[39] M. Durante, private communication

[40] "Certification of the Radiation Resistance of Coil Insulation Material", EuCARD Deliverable Report, EuCARD-Del-D7-2-1-final-1.docx.

[41] A.V. Zlobin, "Status of Nb3Sn Accelerator Magnet R\&D at Fermilab", CERN Yellow Report CERN-2011-003, pp. 50-58.

[42] G. Ambrosio, et al., "Development and Coil Fabrication for the LARP 3.7-m Long Nb3Sn Quadrupole”, IEEE Trans. Appl. Supercond., vol. 19, no. 3, pp. 1231 - 1234, Jun. 2009.

[43] P. Fessia, CERN, private communication.

[44] G. Ambrosio, et al., "Test results of the first $3.7 \mathrm{~m}$ long Nb3Sn quadrupole by LARP and future plans", IEEE Trans. Appl. Supercond., vol. 21, no. 3, pp. , 1858 - 1862, Jun. 2011.

[45] H. Felice, et al., "Impact of Coil Compaction on Nb3Sn LARP HQ Magnet”, IEEE Trans. Appl. Supercond., 224001904.

[46] S. I. Bermudez, G. Ambrosio, R. Bossert, D. Cheng, P. Ferracin, S. Krave, J. C. Perez, J. Schmalzle, M. Yu, "Coil end optimization of the low $\beta$ quadrupole for the High Luminosity LHC", this conference.

[47] S. Caspi, S. Gourlay, R. Hafalia, A. Lietzke, J. O'Neill, C. Taylor, and A. Jackson. "The use of pressurized bladders for stress control of superconducting magnets", IEEE Trans. Appl. Supercond., Vol, 11, No. 1, pp. 2272-2275, 2001.

[48] M. Juchno, G. Ambrosio, M. Anerella, F. Borgnolutti, D. Cheng, H. Felice, P. Ferracin, J. C. Perez, H. Prin, J. Schmalzle, E. Todesco, "Support Structure Design of the Low- $\beta$ Quadrupoles Short Model for the High Luminosity LHC”, this conference.

[49] E. Todesco, "Quench Limits in the next generation of Magnets", WAMSDO 2013, CERN Yellow Report CERN-2013-006, pp.10-16. 
[50] G. Ambrosio, "Maximum allowable temperature during quench in Nb3Sn accelerator magnets", WAMSDO 2013, CERN Yellow Report CERN-2013-006, pp.43-46.

[51] S. Caspi et al., "Measured Strain in Nb3Sn Coils During Excitation and Quench”, IEEE Trans. Appl. Supercond. Vol. 15, No. 2, pp. 1461-1464, 2005.

[52] G. Manfreda, et al., "Quench Protection Study of the Nb3Sn Low-beta Quadrupole for the LHC Luminosity Upgrade”, IEEE Trans. Appl. Supercond., 234700405.

[53] M. Sorbi, G. Ambrosio, G. Chlachidze, H. Felice, M. Marchevsky, V. Marinozzi, E. Todesco, "Study of quench protection for the Nb3Sn lowbeta quadrupole for the LHC luminosity upgrade”, this conference.

[54] H. Felice, LBNL, private communication.

[55] E. Ravaioli, et al., "New, Coupling Loss Induced, Quench Protection System for Superconducting Accelerator Magnets", IEEE Trans. Appl. Supercond., 24500905.

[56] E. Ravaioli, H. Bajas, V. I. Datskov, G. Kirby, H. H. ten Kate, A. P. Verweij, "First Test of CLIQ, the New Coupling Loss Induced Quench Protection System, on a Nb3Sn Magnet”, this conference.

[57] A.V. Zlobin, G. Chlachidze, A. Nobrega, I. Novitski, M. Karppinen, “Quench Protection Studies of 11T Nb3Sn Dipole Coils”, IPAC'2014, Dresden, June 2014.

[58] M. Karppinen, et al., "Status of $11 \mathrm{~T}$ Nb3Sn Dipole Project for the LHC", this conference.

[59] A.V. Zlobin, N. Andreev, E. Barzi, G. Chlachidze, V.V. Kashikhin, A. Nobrega, I. Novitski, D. Turrioni, M. Karppinen, D. Smekens, “Testing of a Single 11T Nb3Sn Dipole Coil using a Magnetic Mirror Structure”, IPAC’2014, Dresden, June 2014.

[60] G. Chlachidze, FNAL, unpublished data.

[61] A.V. Zlobin, et al., "Status of 11 T 2-in-1 Nb3Sn Dipole Development for LHC”, IPAC’2014, Dresden, June 2014. 